\title{
Modelling Social Segregation
}

\section{HARVEY GOLDSTEIN \& PHILIP NODEN}

ABSTRACT This paper proposes a multilevel modelling approach to the analysis of social segregation in schools. Using data on free school meal eligibility it shows that the underlying variation between schools for the period 1994-1999 has increased. It also shows that the change is greater for selective than non-selective local education authorities (LEAs). It is suggested that the approach of this paper can be applied generally to the modelling of social segregation at institution level.

\section{INTRODUCTION}

The primary aim of this paper is to provide a methodological contribution to the analysis of segregation. In addition, it provides a substantive contribution to the debate on social segregation in England's secondary schools. Despite the methodological focus, in order to make the paper accessible to a wider readership we have confined most of the technical information to the Appendix. We begin with a summary of the debate on social segregation in schools. We then suggest that multi-level modelling provides a very useful technique for analysing segregation. We go on to provide an example which involves reanalysis of a dataset used previously by one of the authors (Noden, 2000) that includes data on free school meals eligibility in secondary schools in England from 1994 to 1999. This analysis shows an increase in segregation from 1994 to 1999, with a marked increase in segregation in areas operating selective secondary education systems. It also identifies a far smaller increase in segregation associated with the proportion of schools with control over their own admissions. We conclude with a brief discussion of the possible implications of the findings and of the potential future application of the methods presented in the paper.

\section{THE SEGREGATION DEBATE}

The quasi-market reforms of the secondary education system in England and Wales, from 1988 onwards, set up new incentives and opportunities for schools and parents. Parents were given greater opportunity to choose a school for their children and were supported in making their choices through the publication of examination league tables and OFSTED inspection reports. Meanwhile schools were, in most cases, compelled to admit pupils up to their physical capacity (should there be demand for those places) and were in many cases given control over their own admissions policies and practices. Taking these reforms together it was widely predicted that schools would be increasingly socially stratified. This is because it was thought that more advantaged parents would be better able to negotiate bureaucratic admissions procedures or able to afford to send their children further to school. On the 'supply side' it was suggested that, as 


\section{Oxford Review of Education}

school funding was primarily determined by pupil numbers, schools would be keen to enhance their popularity among parents. In order to achieve this, so the theory went, schools would wish to protect their league table position and therefore seek to attract the pupils most likely to succeed in public examinations-that is, more advantaged pupils. Thus when matching pupils to schools, more advantaged pupils would be better placed to apply to 'better' schools (as evidenced by league tables) and those schools would be only too happy to welcome those advantaged pupils. Of course, the theory summarised above implies many assumptions, only some of which have been subjected to empirical scrutiny. For example, it is not clear that we are right to assume that advantaged parents were not already employing effective means of securing a place at a school of their choice, nor has good evidence been presented on which a judgement could be based. Evidence has, on the other hand, been presented on the range of factors taken into account by parents when choosing schools-and examination results appear to be neither the only nor the foremost criterion.

Nevertheless, it came as a surprise when researchers reported only a slight rise in segregation after 1988 followed by an even steeper fall (Gorard \& Fitz, 1998). Subsequent reports from this same research team suggested that segregation again began to rise from 1997, though never reaching the level reported for 1988 (Gorard et al., 2001). The indicator of disadvantage employed in that research was eligibility for or receipt of free school meals - the most widely used indicator of educational disadvantage in the UK. Broadly speaking, the method for analysing segregation involved identifying the proportion of pupils eligible for free school meals who would have to swap schools with a pupil ineligible for free school meals in order to achieve the same percentage of pupils eligible for free meals at every school in a given area. Gorard and colleagues have reported index scores at the level of the LEA, region and country.

Indices similar to the one employed in that research, and by one of the authors of this paper (Noden, 2000), have a long history in social research (e.g. Duncan \& Duncan, 1955), especially arising from studies of residential segregation in the United States. However, each index has its own limitations and these are well documented (e.g. James \& Taeuber 1985; Massey \& Denton, 1988). Such indices tend to identify a single dimension of segregation which is then measured. In this paper we propose an alternative approach which involves formal statistical modelling of the data.

We would suggest there are three key advantages to our approach. First, and crucially, such an approach does not involve aggregating data to the level of the LEA in such a way that information is lost. Second, while index measures typically use observed proportions, ratios or differences and ignore the role of random variation, a modelling approach involves a probabilistic model. Thus, in the case of index scores, suppose that there is no underlying variation between schools in the proportion of disadvantaged pupils, merely variation in the sense that over time each school will vary randomly about the same overall mean proportion. At any one time, the observed proportions will differ by chance and any index (such as those used by Gorard and Noden) will therefore have a positive value and will also depend intrinsically on variables such as school size.

However, it is when we question how changes vary between areas that we see the substantial advantages of a modelling approach. Here, the approach allows us to have 'random coefficients' in the model, that is, coefficients of predictor variables that vary across areas. We can also allow the between-school or between-area variation to be a function of predictor variable values. This is an interesting analytical innovation. In regression-type analyses variance is often regarded as a nuisance parameter whereas in 
this analysis the variance is the key item of interest. For example, suppose we have two time points, by estimating the variance at the two points (together with the covariance) we can then carry out a test for whether or not the variances are equal at time one and time two. This would directly address the issue of changing segregation over time [1]. Further, if a significant change in segregation is identified in this manner, predictor variables may be added to the model, and in particular to the variance function. This may provide an insight into, for example, what area characteristics are associated with a greater increase in segregation. Such predictor variables might include population density (reflecting greater opportunities for parents to choose between schools) or, as is reported in this paper, what proportion of schools are their own admissions authority. Similarly, we could address the issue of changing between-area segregation over time. A properly formulated model, moreover, allows us to test the assumptions made by the model in order to arrive at a model that closely describes the observed data.

Before presenting an example of this method, it is important to note some limitations of the data. First, the indicator of disadvantage used in this paper, as in much educational research, is eligibility for free school meals. In this case, the distribution of pupils eligible for free meals among schools in different LEAs is examined to illuminate changes in the intakes of those schools. However, only a school level figure is available, rather than a figure for successive Year 7 cohorts. Consequently, even in schools where almost all pupils complete every year of schooling (rather than changing schools at any point), changes in the proportion of pupils eligible for free school meals are not only affected by the characteristics of each new Year 7 cohort but also by the characteristics of school leavers. In addition, it is important to recognise that eligibility for free school meals is not a fixed characteristic of pupils but rather reflects the economic circumstances of their households at a particular moment. Thus changing levels of eligibility for free school meals reflect not only the change in a school's intake (compared with the cohort leaving the school) but also movements in to and out of eligibility-that is changes in the socio-economic circumstances of the pupil group. As has been suggested elsewhere, these changes may mask any shifts in the characteristics of successive cohorts of pupils entering the school (Gibson \& Asthana, 2000; Noden, 2002). In addition, the criteria determining pupils' eligibility for free school meals are modified from time to time as changes are made to the benefits system and unfortunately we are unable to assess the impact of any such changes in different schools. Second, the LEA is used as the proxy for the local competitive market. Of course in reality a list of all the schools in an LEA reflects neither the choice set available to any particular parent nor the secondary schools with which any particular school is competing for pupils.

The dataset consists of some 3560 schools in 128 LEAs for the years 1994-1999 recording the total number on roll and the number eligible for and receiving free school meals. It would be desirable to examine data for the period from 1988 onwards, however, since data relating to eligibility for free school meals (thought to be a better proxy for disadvantage than free school meals receipt) were only collected from 1993 onwards [2]. The LEA boundaries are those in operation in 1998 [3].

\section{MODELLING SECONDARY SCHOOL SEGREGATION, 1994-1999}

Consider the basic structure of educational catchment areas, in this case LEAs, indexed by $j$ and schools within areas indexed by $i$. The response is, in this case, the proportion of disadvantaged pupils in a school measured as the proportion eligible for free school 
meals or, put another way, the probability that a randomly chosen pupil in school $i$ in area $j$ is eligible.

We write a simple variance components model in the standard way (Goldstein, 1995) as

$\log \left[\pi_{i j} /\left(1-\pi_{i j}\right)\right]=\operatorname{logit}\left(\pi_{i j}\right)=(X \beta)_{i j}+v_{j}+u_{i j}$

$p_{i j} \sim \operatorname{bin}\left(n_{i j}, \pi_{i j}\right)$

$v_{j} \sim N\left(0, \sigma_{v}^{2}\right), u_{i j} \sim N\left(0, \sigma_{u}^{2}\right)$

Note that this implies a particular (logistic) scale on which the effects operate [4]. In the present paper we restrict our analyses to the use of this logistic function which is the one most commonly used in the social sciences.

In the first line of the model shown above, the predictor variables, denoted by $\mathrm{X}$, can include time, school type, number of schools in an area etc. The term $v_{j}$ denotes the residual (not explained by the predictor variables) at the area level and $u_{i j}$ the school level residual. The area and school variances of these residuals $\sigma_{v}^{2}, \sigma_{u}^{2}$ are measures of segregation at the area and school-within-area levels respectively; they measure the variation between areas and schools after adjusting for the fixed effects (X). The fixed effect coefficients measure the dependence of the proportion eligible for free school meals on the predictors, for example any dependence upon time. Note that the size of school does not explicitly enter into this model (unless we choose to include it as a predictor), but is used during the statistical estimation procedure. Note also that variation within and between areas is modelled simultaneously. Indices such as that of Gorard (2000) or Noden (2000) attempt to measure the variation at a single spatial scale (for example, between schools within an LEA, or between LEAs within a region). It is quite possible for within area variation to decrease and overall variation to increase if the between area variation increases.

The second and third lines of (1) show the assumptions required by the model. The second line states that the observed proportion based upon the number in the school, $n_{i j}$, has an underlying mean value, $\pi_{i j}$, and it is this underlying proportion that is the focus of interest. The third line indicates the assumption that the residuals are normally distributed. The legitimacy of this assumption is tested and, in the Appendix, shown to be well founded.

We now look at the national data for secondary schools that were also used in the analysis by Noden (2000). (A similar dataset was also used by Gorard (2000).) In the present analysis we first fit model (1) for each year, incorporating variation between LEAs as well as schools with just an intercept term in the fixed part of the model. The resulting between school and between LEA variances are given in Table I. We see a fairly steady increase in the between school variation and an overall upward trend until 1999. However we do not see a consistent change in between LEA variation.

Ideally we would wish to assess the significance of an overall trend, but this is not possible because we only have available the total numbers of pupils in each school in each year, and in successive years most of the children in any one school will be the same ones. What we would like to have are data on the new entrants to each school each year and to base trends upon these data, which would both provide a more sensitive analysis and allow, for example, the construction of interval estimates for trends. Nevertheless, for the two extreme years of 1994 and 1999 it seems reasonable to assume that the data are in fact based upon different groups of pupils and we 
TABLE I. Variance estimates (standard errors) for each year

\begin{tabular}{llll}
\hline Year & Between school & Between LEA & Total \\
\hline 1994 & $0.625(0.016)$ & $0.491(0.066)$ & 1.116 \\
1995 & $0.636(0.016)$ & $0.522(0.072)$ & 1.158 \\
1996 & $0.650(0.016)$ & $0.503(0.064)$ & 1.153 \\
1997 & $0.660(0.017)$ & $0.498(0.069)$ & 1.158 \\
1998 & $0.685(0.017)$ & $0.506(0.068)$ & 1.191 \\
1999 & $0.691(0.017)$ & $0.506(0.068)$ & 1.197 \\
\hline
\end{tabular}

These models and subsequent ones are fitted using PQL2 estimation in the MLwiN software package (Rasbash et al., 2000). We have checked the fits using MCMC with diffuse priors and obtain substantially the same results.

therefore fit the following model for these two years, using the observed proportions eligible for free school meals in each year, and this is represented in Model (2).

$$
\begin{aligned}
& \log \left[\pi_{1 i j} /\left(1-\pi_{1 i j}\right)\right]=\operatorname{logit}\left(\pi_{1 i j}\right)=\beta_{1}+v_{1 j}+u_{1 i j} \text { for } 1994 \\
& \log \left[\pi_{2 i j} /\left(1-\pi_{2 i j}\right)\right]=\operatorname{logit}\left(\pi_{2 i j}\right)=\beta_{2}+v_{2 j}+u_{2 i j} \text { for } 1999 \\
& p_{h i j} \sim \operatorname{bin}\left(n_{h i j,} \pi_{h i j}\right) h=1,2 \\
& \left(\begin{array}{l}
v_{1} \\
v_{2}
\end{array}\right) \sim N\left(\begin{array}{cc}
\sigma_{v 1}^{2} & \\
\sigma_{v 12} & \sigma_{v 2}^{2}
\end{array}\right) \\
& \left(\begin{array}{l}
u_{1} \\
u_{2}
\end{array}\right) \sim N\left(\begin{array}{cc}
\sigma_{u 1}^{2} & \\
\sigma_{u 12} & \sigma_{u 2}^{2}
\end{array}\right)
\end{aligned}
$$

The subscripts 1 and 2 refer to 1994 and 1999 respectively. The terms $\beta_{1}, \beta_{2}$ represent the intercepts for each year; $v_{1 j}, v_{2 j}$ represent the area residuals for each year; and $u_{1 i j}$, $u_{2 i j}$ represent the school residuals for each year. At the area and school levels we have a variance for each year and a covariance between years. The results are given in Table II which is included in the Appendix.

The hypothesis tests shown in Table II indicate that the between school variance was significantly larger in 1999 than in 1994 whereas the increase in between LEA variation was not statistically significant. It is important to note that the estimate of extra-binomial variation suggests that there may be further hierarchical levels in the data, such as the existence of relatively homogeneous areas within LEAs with underlying variation between these, or alternatively that there may be important predictor variables that have been omitted.

The data show clearly that within LEAs the between school variability has increased over the period 1994-1999, with a suggestion that the same has occurred, but less markedly, for the between LEA variability. We need to remember that grouping schools by LEA is not an entirely satisfactory way of accounting for the schools actually available to a child in that area. This analysis provides a baseline description of changes in segregation and we now explore how these changes are affected by other factors. 


\section{AREA CHARACTERISTICS AND THE INCREASE IN SEGREGATION}

In this section we extend the model to investigate what kinds of area characteristics are associated with a greater increase in segregation. Specifically we examine the importance of whether an LEA includes selective schools and also the importance of the proportion of schools with control over their own admissions arrangements.

We first elaborate the model by using LEA information on whether schools are selective, using their status in 1998. Only 32 LEAs had such schools and so we have limited our analysis to whether or not there are any such schools within the LEA (rather than, for example, the proportion of pupils attending selective schools). The model, shown as a single equation rather than separate equations for the two years, is an extension of the previous one and is shown as model (3) in the Appendix.

The fixed part of the model, the results from which are shown in the upper section of Table III in the Appendix, models the mean level of eligibility for free school meals in 1994 and 1999, together with the differences in each year between schools located in LEAs without selective schools and those in LEAs which include selective schools. We see that there is a considerably smaller percentage of FSM eligible children in those LEAs with selective schools (0.49 on the logistic scale) and this is no surprise as grammar schools tend to be located in more affluent LEAs. For 1994 the median percentages for schools in areas with and without selective schools are $15.4 \%$ and $24.8 \%$. For 1999 these are respectively $15.1 \%$ and $24.1 \%$. These changes are not significantly different for the two types of areas (although the proportion eligible did in fact rise and then fall within this period). The between school variances are, however, considerably larger for the selective LEAs at both occasions. Again, this is not surprising as segregation between schools is greater in areas operating the eleven plus-disadvantaged pupils are less likely to be successful in this entrance examination and are therefore more likely to be concentrated in secondary modern schools. More interestingly, for both types of LEA there is a highly significant increase between the years, as we found in Table II. Furthermore, as the significance test in Table III shows, the change in variance between 1994 and 1999 is greater for the selective LEAs than for the non-selective ones; in other words segregation increased between these years and increased more among those LEAs which contain selective schools.

We now look at the effect of having schools which have control over their own admission policies. It was suggested earlier that devolved control over school admissions might facilitate greater social segregation. The number of schools with control over admissions - that is, Grant Maintained or Voluntary Aided schools-rose from 919 in 1994 to 1017 in 1999 [5]. For each LEA we have the percentage of schools with such control, again as measured in 1998. In that year some $29 \%$ of English secondary schools were responsible for their own admissions. We now fit a model in which the percentage in each LEA is an additional predictor (in the fixed part of the model) and the between-school variance is allowed to be a (quadratic) function of this percentage. The fitted model is shown as model (4) in the Appendix and includes a random coefficient for admission percentage. We present the results in Table IV, also shown in the Appendix, and describe them visually as Figure 1 by plotting the variance as a function of admission percentage and selectivity.

In effect, in the reformulated model, two new terms are added to the fixed part of the model $\left(\beta_{12} x_{12 j}\right.$ and $\left.\beta_{22} x_{22 j}\right)$ representing the proportion of schools with control over admissions (measured in 1998) with their coefficients being the slopes of the regression 


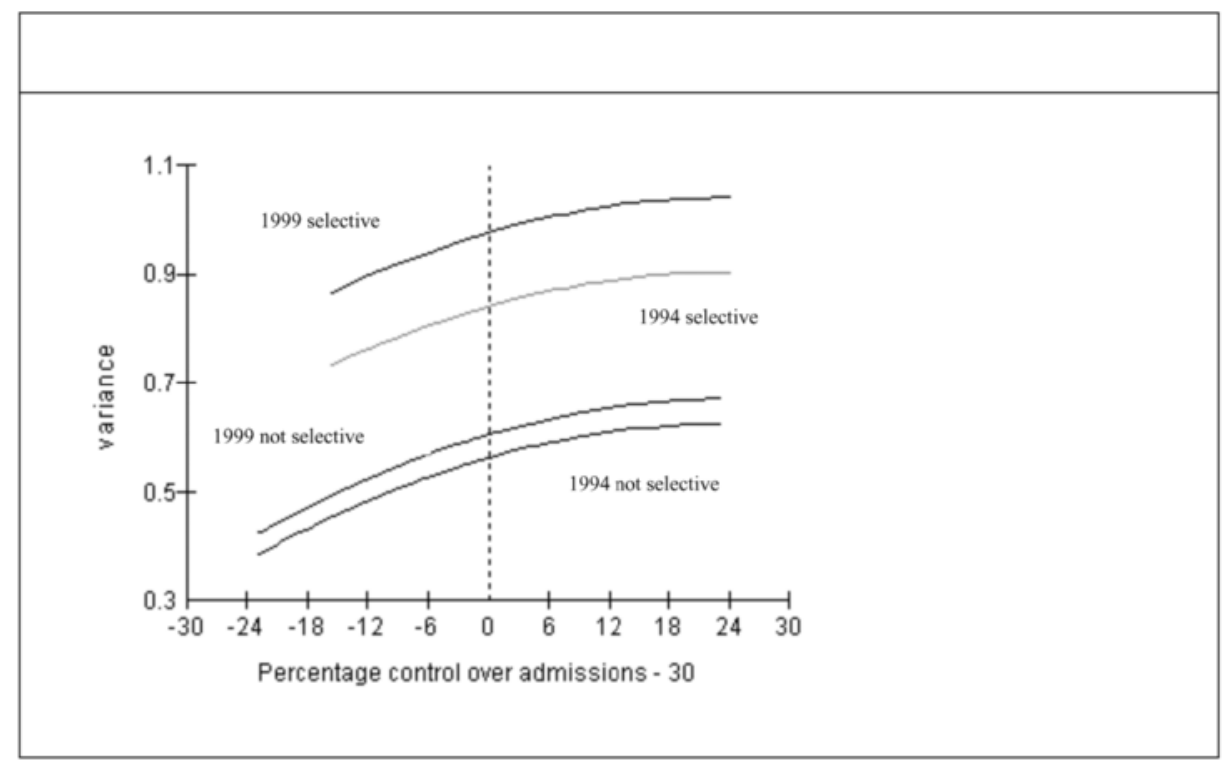

FIG. 1. Between-school variance for selective and non-selective LEAs in 1994 and 1999 by percentage admission controlling schools. Estimates used from Table IV.

lines for 1994 and 1999 respectively. The other additional term $\left(u_{12 i j} x_{2 j}\right)$ allows the between-school variation to be a function of the admission percentage.

The extra binomial parameter here is as it was for model (3), 1.52. The estimates for selective and non-selective variances and their changes over time are very similar to those in Table III. The average increase for a $10 \%$ increase in the percentage of schools with control over admissions is approximately a tenth of the increase associated with having selective schools. Nevertheless, there $i$ s an increase in variance with increasing percentage of admission controlling schools, which is similar for selective and nonselective areas (reported in Table IV as the linear coefficients of 0.0026 and 0.0025 respectively). We note that in the fixed part of the model the effect of percentage admission control on the proportion of free school meal eligible pupils is small and of borderline $(5 \%)$ significance. (This suggests that schools with control over their own admissions are marginally more likely to be located in more disadvantaged areas.) Nevertheless, the proportion of schools with control over admissions still affects the between-school variation. Figure 1 shows the plots of the between-school variance for selective and non-selective LEAs in 1994 and 1999 by percentage admission controlling schools.

We see that there is less change in variance with increasing control over admissions as the percentage with such control increases, and the difference between the years and the selective and non selective LEAs is clear.

\section{CONCLUDING REMARKS}

The analyses shown above suggest that, from 1994 to 1999, there was a fairly smooth increase in the between-school variance in levels of free school meals eligibility and, more importantly, a significant increase from the first year to the last year for which we 


\section{Oxford Review of Education}

have data. Social segregation was found to be substantially greater in areas which included at least some selective secondary schools. This is, of course, not a surprise: socio-economically advantaged pupils are more likely to pass the eleven plus and thus the examination acts as a sorting mechanism for socio-economic status. Of greater interest is the finding that, from 1994 to 1999, between-school variance increased more in selective LEAs than in non-selective areas.

This finding provides us with something of a puzzle. It might reasonably be suggested that selective LEAs are the areas in which we would least expect to see an increase in segregation arising from the quasi-market reforms. These areas, after all, already had a highly effective means of producing socially segregated schools and we would hardly expect the segregating effects of this selection process to be enhanced by the quasimarket reforms.

One interpretation that we may tentatively pose is that changing levels of social segregation, as measured by the free school meals indicator, may be related to overall levels of poverty. As was pointed out earlier, the distribution of pupils eligible for free school meals is determined not only by the allocation of pupils between secondary schools when they enter in Year 7 or change schools subsequently (net of those leaving at the end of their school career). It also reflects pupils moving into or out of eligibility for free school meals as, for example, their parents move on to or off benefits. Thus it also reflects the distribution of the disposition to move on or off benefits. And this disposition may be distributed neither evenly nor randomly. For example, we might postulate that, in a period of economic growth, those parents best placed to take advantage of new employment opportunities will be the first to move off benefits and, further, that those best placed may be more concentrated in relatively advantaged schools. Returning to the case of greater increases in segregation in selective areas, it may be that the eleven plus is effective not only in selecting higher attaining children but also, even among those eligible for free school meals, in identifying those households more likely to move off benefits. Further, such a process, in a more moderated form, may be at work in non-selective areas. (Indeed, there is an apparent inverse relationship between the levels of segregation reported by Gorard and colleagues (Gorard et al., 2001) and levels of free school meals take up during the same period (Noden, 2002).) This is, of course, a speculative hypothesis. However, it is reminiscent of research which suggested that income and eligibility for state benefits are a less than adequate means of identifying social class and may mask differences in cultural capital or family circumstances (Edwards et al., 1989). Eligibility for free school meals is of course an extremely crude indicator of socio-economic status and a more sensitive measure may yield a more illuminating picture of changing levels of social segregation.

In conclusion, we would suggest that the methods presented in this paper offer a promising avenue for further analysis of segregation, particularly if deployed with a more stable dependent variable. In addition, these models can be applied to measure 'achievement segregation' using readily available examination and Key Stage test data.

\section{NOTES}

[1] For more time points we would set up a 'repeated measures' model with 'occasion' (time point) as a further level within schools. 
[2] Data relating to 1993 were not included in the dataset because of matching problems.

[3] Data for Wakefield and Doncaster have been excluded because both authorities underwent a transition from a three-tier education to a two-tier system during this period.

[4] We could choose an alternative function linking the linear predictor on the right hand side of (1) to the probability $\pi_{i j}$ of a pupil in school $i$ in area $j$ being eligible for free school meals. Examples are the probit and complementary log-log functions (see, for example, McCullagh \& Nelder, 1989). It will often be useful to try different functions in a sensitivity analysis.

[5] In 1989 there were only 521 Voluntary Aided Secondary schools. Some schools gained Grant Maintained status the following year, which was replaced in 2000 by Foundation status. In 2002 there were 1050 secondary schools with control over their own admissions arrangements, representing some $30 \%$ of schools.

\section{REFERENCES}

Duncan, O. \& Duncan, B. (1955) A methodological analysis of segregation indexes, American Sociological Review, 20, pp. 210-217.

Edwards, T., Fitz, J. \& WhitTy, G. (1989) The State and Private Education: an evaluation of the Assisted Places Scheme (Falmer, Basingstoke).

Gibson, A. \& Asthana, S. (2000) What's in a number? Research Papers in Education, 15, 2, pp. 133-154.

GoldsteIn, H. (1995) Multilevel Statistical Models (London, Edward Arnold; New York, Wiley).

Gorard, S. (2000) Education and Social fustice (Cardiff, University of Wales Press).

Gorard, S. \& FITZ, J. (1998) The more things change ... the missing impact of marketisation? British fournal of Sociology of Education, 19, pp. 365-376.

Gorard, S., Fitz, J. \& TAYLOR, C. (2001) School choice impacts: what do we know? Educational Researcher, 30, 7, pp. 18-23.

JAmes, D. \& TAEuber, K. (1985) Measures of segregation, in: N. Tuma (Ed.) Sociological Methodology (San Fransisco, CA, Jossey-Bass), pp. 1-32.

Massey, D. \& Denton, N. (1988) Dimensions of residential segregation, Social Forces, 67, pp. 281-315.

McCullagh, P. \& Nelder, J. (1989) Generalised Linear Models (London, Chapman).

Noden, P. (2000) Rediscovering the impact of marketisation: dimensions of social segregation in England's secondary schools, 1994-99, British Fournal of Sociology of Education, 21, 3, pp. 371-390.

Noden, P. (2002) Education markets and social polarization: back to square one? Research Papers in Education, 17, 4.

Nuttall, D. L., Goldstein, H., Prosser, R. \& Rasbash, J. (1989) Differential school effectiveness, International fournal of Educational Research, 13, pp. 769-776.

Rasbash, J., Browne, W., Goldstein, H., Yang, M. et al. (2000) A User's Guide to MlwiN, 2nd edn (London, Institute of Education).

Correspondence: Professor Harvey Goldstein, Institute of Education, 20 Bedford Way, London WC1H 0AL, UK. Email: h.goldstein@ioe.ac.uk 


\section{Oxford Review of Education}

\section{APPENDIX}

a) Table II and Figure 2

Note: the estimates differ slightly from those shown in Table I because this is the result of a joint analysis of both years.

TABLE II. Fitting a model for years 1994 and 1999

\begin{tabular}{llc}
\hline Fixed & Estimate & S.E. \\
\hline Intercept 1994 & -1.553 & 0.064 \\
Intercept 1999 & -1.585 & 0.066 \\
Random & & \\
$\sigma_{u 1}^{2}$ (School level variance 1994) & 0.636 & 0.016 \\
$\rho_{u 12}$ (Correlation between school residuals for 1994 and 1999) & 0.95 & \\
$\sigma_{u 2}^{2}$ (School level variance 1999) & 0.707 & 0.017 \\
$\sigma_{v 1}^{2}$ (LEA level variance 1994) & 0.490 & 0.066 \\
$\rho_{v 12}$ (Correlation between LEA residuals for 1994 and 1999) & 0.98 & \\
$\sigma_{v 2}^{2}$ (LEA level variance 1999) & 0.508 & 0.069 \\
\hline
\end{tabular}

Hypothesis tests: $\sigma_{u 1}^{2}=\sigma_{u 2}^{2} ; \chi_{1}^{2}=67.0 \mathrm{P}<0.001 . \sigma_{v 1}^{2}=\sigma_{v 2}^{2} ; \chi_{1}^{2}=0.87 \mathrm{P}<0.10$.

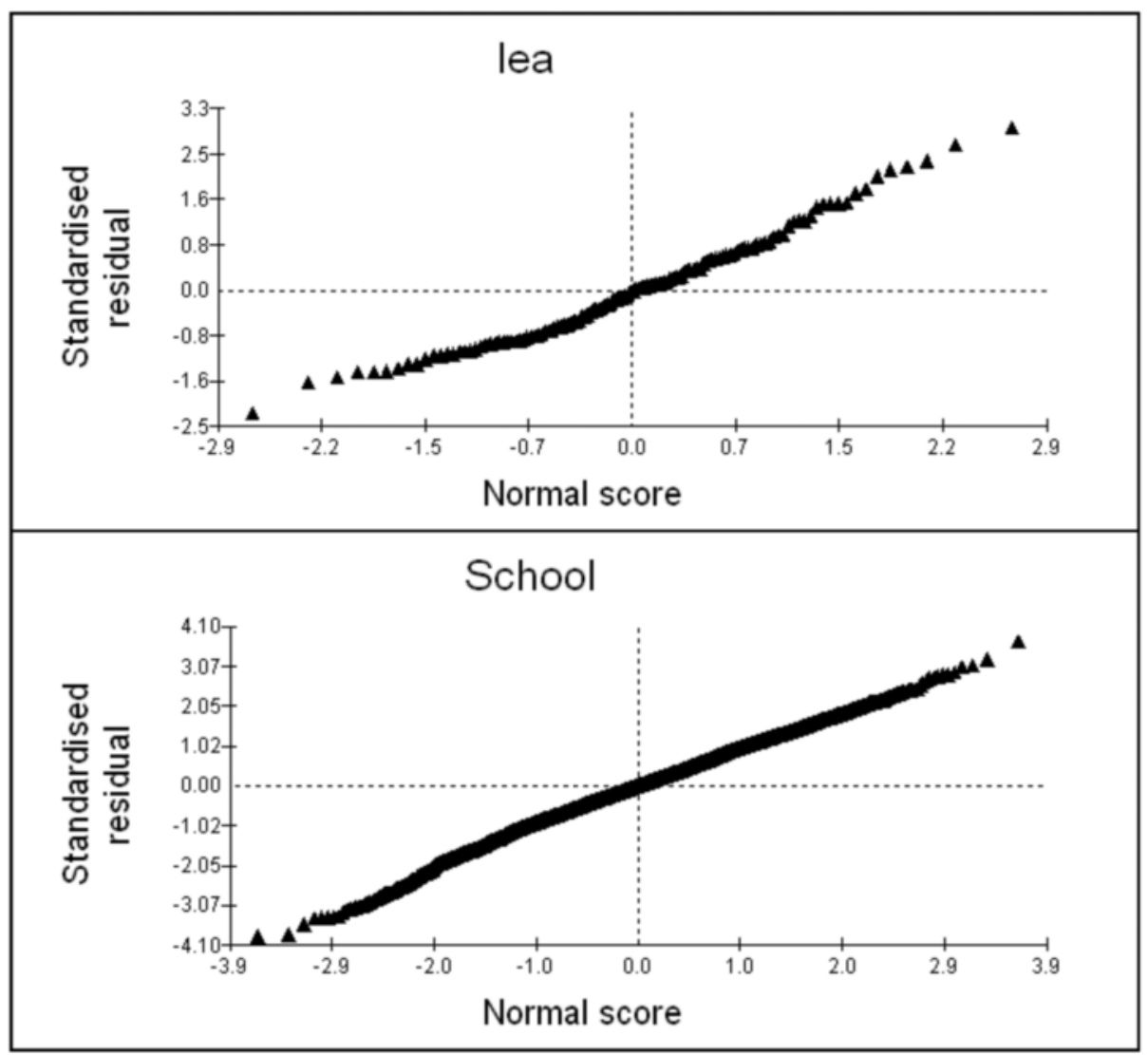

FIG. 2. Residual plots for schools and LEAs in 1994. 
We have also studied the distribution of the residuals from these analyses and they appear satisfactorily Normal, although there is some slight evidence of non-normality for the LEA residuals. The school level and LEA level residuals for 1994 are shown in Figure 2.

In this analysis, because we have (up to) two responses for each school we can obtain an independent estimate of the within school variation, which allows us to estimate 'extra-binomial' variation. What we mean by this is that in the standard model formulation we assume that the observed proportion $p_{i j}$ has a binomial distribution with mean $\pi_{i j}$, as stated in (1). In some cases, however, such an assumption may be unreasonable, for example if there is an extra hierarchical level in the model which has been omitted (see Goldstein, 1995, for further details). When this is done we find some evidence for such variation, with a coefficient of 1.68 (this will be 1.0 if the variation is exactly binomial). This suggests that there may indeed be further hierarchical levels in the data, such as the existence of relatively homogeneous areas within LEAs with underlying variation between these, or alternatively that there may be important predictor variables that have been omitted.

b) Model (3) and Table III

$$
\begin{aligned}
& \log \left[\pi_{i j} /\left(1-\pi_{i j}\right)\right]=\operatorname{logit}\left(\pi_{i j}\right) \\
& =\beta_{10} x_{10 j}+\beta_{20} x_{20 j}+\beta_{11} x_{11 j}+\beta_{21} x_{21 j}+v_{1 j}+v_{2 j}+u_{10 i j} x_{10 j}+u_{20 i j} x_{20 j}+u_{11 i j} x_{11 j}+u_{21 i j} x_{21 j} \\
& p_{i j} \sim \operatorname{bin}\left(n_{i j}, \pi_{i j}\right) \\
& \left(\begin{array}{l}
v_{1} \\
v_{2}
\end{array}\right) \sim N\left(\begin{array}{cc}
\sigma_{v 1}^{2} & \\
\sigma_{v 12} & \sigma_{v 2}^{2}
\end{array}\right) \\
& \left(\begin{array}{l}
u_{10} \\
u_{20} \\
u_{11} \\
u_{21}
\end{array}\right) \sim N\left(\begin{array}{llll}
\sigma_{u 10}^{2} & & & \\
\sigma_{u 120} & \sigma_{u 20}^{2} & & \\
& & \sigma_{u 11}^{2} & \\
& & \sigma_{u 121} & \sigma_{u 21}^{2}
\end{array}\right)
\end{aligned}
$$

where

$x_{10 j}=1$ if response is for 1994 and there are no selective schools, otherwise 0

$x_{20 j}=1$ if response is for 1999 and there are no selective schools, otherwise 0

$x_{11 j}=1$ if response is for 1994 and there are no selective schools, otherwise 0

$x_{21 j}=1$ if response is for 1999 and there are no selective schools, otherwise 0

In this model the school level residuals $u_{10 i j}, u_{20 i j}, u_{11 i j}, u_{21 i j}$ are respectively those for non-selective LEAs at occasions 1 and 2 and selective LEAs at occasions 1 and 2. The school level covariance matrix consists of two separate blocks; the upper left $2 \times 2$ block describes the covariance matrix for the non-selective LEAs for 1994 and 1999 and the lower right block describes the covariance matrix for the selective LEAs for 1994 and 1999.

Fitting extra binomial variation gives a value of 1.52, an improvement on the previous model and indicating a better specified model structure. (We have investigated the use of probit and log-log link functions and the conclusions remain substantially the same.) 
TABLE III. Fitting a model for years 1994 and 1999, including selective/non selective LEAs

\begin{tabular}{lll}
\hline Fixed & Estimate & S.E. \\
\hline Non-selective intercept 1994 & -1.391 & \\
Non-selective intercept 1999 & -1.424 & \\
Selective-non-selective intercept 1994 & -0.492 & 0.155 \\
Selective-non-selective intercept 1999 & -0.489 & 0.159 \\
Random & & \\
$\sigma_{u 10}^{2}$ (variance of 1994 school residuals, non-selective LEAs) & 0.505 & 0.016 \\
$\rho_{u 120}(1994 \& 1999$ school residuals correlation, non-selective LEAs) & 0.94 & \\
$\sigma_{u 20}^{2}$ (variance of 1999 school residuals, non-selective LEAs) & 0.547 & 0.018 \\
$\sigma_{u 11}^{2}$ (variance of 1994 school residuals, selective LEAs) & 0.865 & 0.047 \\
$\rho_{u 121}$ (1994 \& 1999 school residuals correlation, selective LEAs) & 0.96 & \\
$\sigma_{u 21}^{2}$ (variance of 1999 school residuals, selective LEAs) & 1.035 & 0.056 \\
$\sigma_{v 1}^{2}$ (variance of 1994 LEA residuals) & 0.463 & 0.065 \\
$\rho_{v 12}$ (1994 \& 1999 LEA residuals correlation) & 0.98 & \\
$\sigma_{v 2}^{2}$ (variance of 1999 LEA residuals) & 0.482 & 0.068 \\
\hline
\end{tabular}

Test hypothesis symbol $H_{0}: \sigma_{u 20}^{2}-\sigma_{u 10}^{2}=\sigma_{u 21}^{2}-\sigma_{u 11}^{2} ; \chi_{1}^{2}=21.6, \mathrm{P}<0.001$.

The data do not allow for the independent estimation of all 6 school level parameters in (3), and in fact only 5 have been directly estimated, the covariance between the occasions for the selective LEAs being a complex function of these 5, namely $\sigma_{u 120}+\left(\sigma_{u 11}^{2}+\sigma_{u 21}^{2}-\sigma_{u 10}^{2}-\sigma_{u 20}^{2}\right) / 2$.

c) Model (4) and Table IV

$$
\begin{gathered}
\log \left[\pi_{i j} /\left(1-\pi_{i j}\right)\right]=\operatorname{logit}\left(\pi_{i j}\right)=\beta_{10} x_{10 j}+\beta_{20} x_{20 j}+\beta_{11} x_{11 j}+\beta_{21} x_{21 j}+\beta_{12} x_{12 j}+\beta_{22} x_{22 j}+v_{1 j} \\
+v_{2 j}+u_{10 i j} x_{10 j}+u_{20 i j} x_{20 j}+u_{11 i j} x_{11 j}+u_{21 j} x_{21 j}+u_{12 i j} x_{2 j}
\end{gathered}
$$

$p_{i j} \sim \operatorname{bin}\left(n_{i j}, \pi_{i j}\right)$

$\left(\begin{array}{l}v_{1} \\ v_{2}\end{array}\right) \sim N\left(\begin{array}{cc}\sigma_{v 1}^{2} & \\ \sigma_{v 12} & \sigma_{v 2}^{2}\end{array}\right)$

$\left(\begin{array}{l}u_{10} \\ u_{20} \\ u_{11} \\ u_{21} \\ u_{12}\end{array}\right) \sim N\left(\begin{array}{lllll}\sigma_{u 10}^{2} & & & & \\ \sigma_{u 120} & \sigma_{u 20}^{2} & & & \\ & & \sigma_{u 11}^{2} & & \\ & & \sigma_{u 1121} & \sigma_{u 21}^{2} & \\ \sigma_{u 1012} & \sigma_{u 2012} & \sigma_{u 112} & \sigma_{u 2112} & \sigma_{u 12}^{2}\end{array}\right)$

The additional terms in this model are:

$x_{2 j}=$ percentage of schools with admission control in 1998

$x_{12 j}=$ percentage of schools with admission control in 1998 \& response is 1994 proportion

$x_{22 j}=$ percentage of schools with admission control in 1998 \& response is 1999 proportion

This is a convenient and parsimonious representation of the model (see Goldstein, 1995, chapter 3). The form of the between-school covariance matrix in (4) implies that the between-school variance is a function of selectivity, as in the model given by (3), together with a quadratic function of the admission percentage. 
TABLE IV. Fitting a model for years 1994 and 1999, including selective/non selective LEAs and admission control percentage (measured around its approximate mean value of 30\%). The 1994 and 1999 fixed coefficients for the latter are virtually identical and only the common value is shown

\begin{tabular}{|c|c|c|}
\hline Fixed & Estimate & S.E. \\
\hline Non-selective intercept 1994 & -1.386 & \\
\hline Non-selective intercept 1999 & -1.418 & \\
\hline Selective-non-selective intercept 1994 & -0.568 & 0.161 \\
\hline Selective-non-selective intercept 1999 & -0.564 & 0.165 \\
\hline$\%$ Admission control & 0.0052 & 0.0033 \\
\hline \multicolumn{3}{|l|}{ Random } \\
\hline$\sigma_{u 10}^{2}$ (variance of 1994 school residuals, non-selective LEAs) & 0.564 & 0.022 \\
\hline$\rho_{u 120}(1994 \& 1999$ school residuals correlation, non-selective LEAs) & 0.95 & \\
\hline$\sigma_{u 20}^{2}$ (variance of 1999 school residuals, non-selective LEAs) & 0.606 & 0.024 \\
\hline \multicolumn{3}{|l|}{$\sigma_{u 1012}$ (linear admissions coefficient in the variance function for } \\
\hline$\sigma_{u 11}^{2}($ variance of 1994 school residuals, selective LEAs) & 0.863 & 0.050 \\
\hline$\rho_{u 1121}(1994 \& 1999$ school residuals correlation, selective LEAs) & 0.95 & \\
\hline$\sigma_{u 21}^{2}$ (variance of 1999 school residuals, selective LEAs) & 1.032 & 0.059 \\
\hline \multicolumn{3}{|l|}{$\sigma_{u 1112}$ (linear admissions coefficient in the variance function for selective } \\
\hline$\sigma_{u 12}^{2}$ (quadratic parameter in the variance function for admissions) & -0.00011 & 0.00003 \\
\hline$\sigma_{v 1}^{2}($ variance of 1994 LEA residuals) & 0.448 & 0.063 \\
\hline$\rho_{v 12}(1994 \& 1999$ LEA residuals correlation) & 0.98 & \\
\hline$\sigma_{v 2}^{2}$ (variance of 1999 LEA residuals) & 0.470 & 0.067 \\
\hline
\end{tabular}

Test hypothesis of no school level percentage admission variation. symbol $\chi_{3}^{2}=33.3 ; \mathrm{P}<0.01$.

For simplicity we have not distinguished between the 1994 and 1999 responses for the random coefficient $u_{12 i j}$ of the 1998 admission control percentage in (4). This means that there is a common parameter, conventionally described as a 'variance' term $\left(\sigma_{u 12}^{2}\right)$ and the respective parameters ('covariances') are equal $\left(\sigma_{u 1012}=\sigma_{u 2012} ; \sigma_{u 2112}=\sigma_{u 1112}\right)$. Thus, effectively we are fitting $6+3=9$ separate random parameters at the school level. The negative 'variance' estimate is not interpreted as a variance in the usual sense since the predictor variable is defined at the higher level of the LEA. Rather it allows us to express the between-school variance as a complex function of this percentage. 
